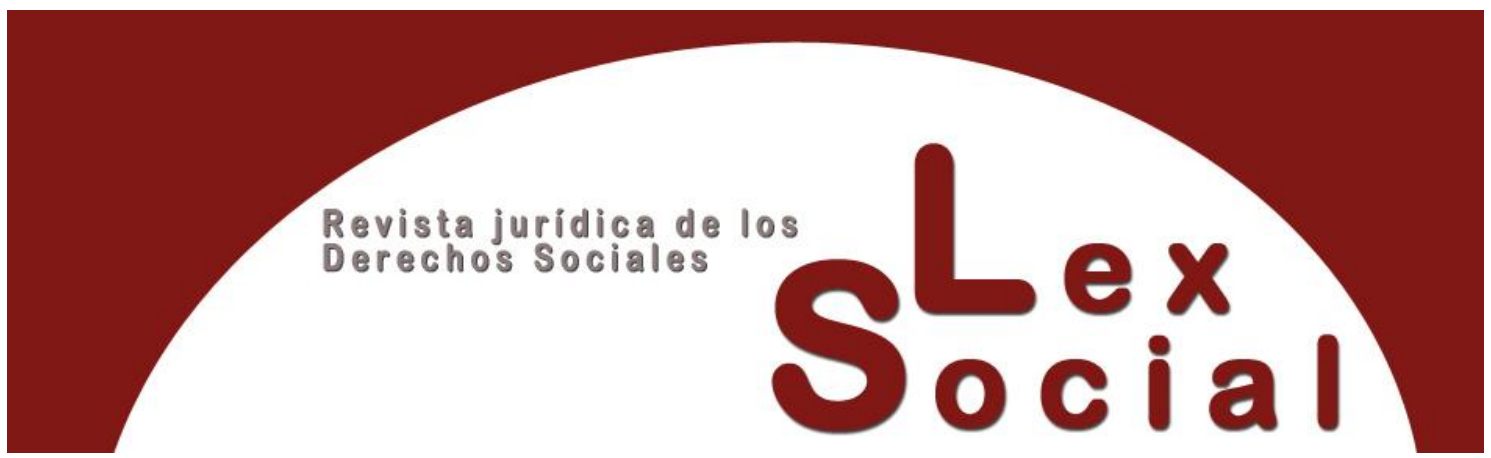

\title{
LA FINA LÍNEA ENTRE EL CUIDADOR NO PROFESIONAL Y EL CUIDADOR PROFESIONAL DE LAS PERSONAS EN SITUACIÓN DE DEPENDENCIA ANTES Y DESPUES DEL COVID-19
}

THE FINE LINE BETWEEN THE NON-PROFESSIONAL CAREGIVER AND THE PROFESSIONAL CAREGIVER OF PERSONS IN A SITUATION OF DEPENDENCE BEFORE AND AFTER COVID-19

PRISCILA MARTÍN VALES

Profesora Asociada Universidad de Salamanca https://orcid.org/0000-0002-3929-1824

Cómo citar este trabajo: Martín Vales, P. (2021). La fina línea entre el cuidador no profesional y el cuidador profesional de las personas en situación de dependencia antes y después del COVID-19. Lex Social: Revista De Derechos Sociales, 11(1), 637-653. https://doi.org/10.46661/lexsocial.5508

\section{RESUMEN}

La figura del cuidador no profesional de personas dependientes ha sido delimitada por el legislador recientemente, sin embargo, todavía queda mucho camino por andar. Las actividades de cuidado y en mayor medida las no profesionales, han recaído de forma mayoritaria en las mujeres, disminuyendo y, en su caso, suprimiendo los derechos sociales de los que gozan los cuidadores profesionales. Este sector claramente feminizado se ha visto incrementado exponencialmente a raíz de la crisis sanitaria provocada por la pandemia Covid-19, además de suspenderse las reformas para que dicha figura obtuviera una mayor necesidad de regulación y protección social.

\section{(cc) BY-NC-SA}


Palabras Clave: convenio especial, cuidador no profesional, seguridad social, protección social, Covid-19.

\begin{abstract}
The figure of the non-professional caregiver of dependent persons has been delimited by the legislator recently, however, there is still a long way to go. Care activities, and to a greater extent non-professional ones, have fallen mostly on women, reducing and, where appropriate, suppressing the social rights enjoyed by professional caregivers.

This clearly feminized sector has been exponentially increased as a result of the health crisis caused by the Covid-19 pandemic, in addition to suspending reforms so that this figure would obtain a greater need for regulation and social protection.
\end{abstract}

KEYWORDS: special agreement, non-professional caregiver, social security, social protection, Covid-19.

SUMARIO

\title{
I. Introducción
}

II. Aproximación al concepto de la figura del cuidador no profesional.

II. Mecanismos de protección de los cuidadores no profesionales de las personas en situación de dependencia

1. Recuperación de la financiación pública.

2. Mecanismos de conciliación y flexibilización.

III. Aumento de la desprotección social tras el inicio de la pandemia Covid-19.

Bibliografía.

\section{Introducción}

La figura del cuidador no profesional se enmarca dentro de la concepción de trabajo humano no remunerado, existiendo en la mayoría de los supuestos una relación de consanguinidad o afinidad dentro de los grados más altos. Así mismo a dichos cuidadores no profesionales se les denomina, también, como «cuidadores informales ${ }^{1}{ }$.

\footnotetext{
${ }^{1}$ Ver VILLABA SÁNCHEZ, Alicia, "Los derechos de los trabajadores con personas de avanzada edad a su cargo. Una aproximación al cuidado de personas de avanzada edad desde el Derecho del Trabajo Español”, Revista Española de Derecho del Trabajo, 2017, n 195.
} 
Sin embargo, existen multitud de razones por las cuales los cuidadores no profesionales asumen este rol. Implicando, el mismo, una renuncia a una prestación laboral total o parcial -mediante un contrato de trabajo a tiempo parcial-, conllevando, por ende, a una falta de cumplimientos legales para adquirir prestaciones sociales de carácter contributivo.

El deber de cuidado y la concepción social de asignación del rol de dicho deber, ha conllevado a que, en la actualidad, este rol lo asuman mayoritariamente las personas de sexo femenino. Así mimo se observa que tanto en los cuidadores no profesionales de personas en situación de dependencia, como en las profesiones en las que rige el deber de cuidado hay una prevalencia notable y destaca de mujeres.

\section{Aproximación al concepto de la figura del cuidador no profesional}

Para poder abordar la figura del cuidador no profesional de personas en situación de dependencia, tendremos que acudir, en primer lugar a la definición legal del cuidado no profesional, la cual se contempla en el artículo 2.5 de la Ley 39/2006 de Promoción de la autonomía Personal y Familiar de las Personas Dependientes (de ahora en adelante Ley 39/2006), estableciendo «la atención prestada a las personas en situación de dependencia en su domicilio, por personas de la familia o de su entorno, no vinculadas a un servicio de atención profesionalizada». Por lo cual, el cuidador no profesional se ocupará de «la atención, compañía y cuidado de otro que, por razón de edad, de enfermedad o discapacidad, no tenga autonomía plena ${ }^{2} \gg$.

Por lo cual, el legislador al incluir en dicho concepto a las personas de la familia o de su entorno, incluye a las relaciones familiares, y a las relaciones de amistad o vecindad. Siendo su aspecto más relevante que los cuidados se presten en el domicilio de la persona dependiente y no gocen de la característica de profesional. En dichos supuestos quedan excluidos los familiares cuyo grado de parentesco supere el tercer grado, haya o no convivencia de la persona en situación de dependencia -esta última consideración la analizaremos posteriormente-.

Las Comunidades Autónomas pueden establecer requisitos adicionales para considerar a una persona como cuidador no profesional, siendo los más utilizados: ser mayor de 18 años; residir en España, o estar empadronado en la Comunidad autónoma correspondiente, en su caso; además, de determinadas condiciones de idoneidad para prestar el cuidado y atención de forma adecuada y no estar vinculado a un servicio de atención profesionalizada, pudiendo establecer tales servicios de cuidados durante un periodo mínimo de tres meses ${ }^{3}$.

\footnotetext{
${ }^{2}$ SELMA PEÑALVA, Alejandra, "La (des)protección social de los cuidadores no profesionales", Revista Derecho social y empresa, 2019, $\mathrm{n}^{\circ} 10$, p. 6.

${ }^{3}$ FERNÁNDEZ COLLADOS, $\mathrm{M}^{\mathrm{a}}$ Belén, "Los cuidados del entorno familiar más de una década después: los vaivenes en su regulación, su aplicación por la doctrina judicial y sus retos en materia de igualdad", Revista Española de Derecho del Trabajo, 2018, n² 210, p. 6.
} 
En muchos casos estas personas dependientes son de avanzada edad, los cuales poseen rentas insuficientes para poder sufragar los costes de los honorarios de los cuidadores profesionales.

En contraposición con lo anteriormente mencionado, la figura del cuidador profesional si se encuentra definido por la Ley 39/2009, considerando dicha figura, en su artículo 2.6, como «los prestados por una institución pública o entidad, con y sin ánimo de lucro, o profesional autónomo entre cuyas finalidades se encuentre la prestación de servicios a personas en situación de dependencia, ya sean en su hogar o en un centro». No pretendiendo dotar dicha norma de un régimen jurídico completo ${ }^{4}$.

En consecuencia podemos advertir que en ocasiones la línea divisoria del cuidador no profesional y la del cuidador profesional de personas en situación de dependencia se difumina hasta su impercepción. Así mimo, podemos encontrarnos en supuestos en los que esta figura del cuidador no profesional adquiera la forma de relación laboral especial de empleados del hogar. No obstante, los empleados del hogar, mayoritariamente mujeres, se asemejan a los cuidadores no profesionales en que los primeros prestan atención y cuidados en el domicilio y no se encuentra vinculada a un servicio de atención profesionalizada 5 .

Después de la entrada en vigor de la Ley 39/2006 y, por ende, de la introducción de la figura del cuidador no profesional, esta se aproximó al trabajador dependiente y por cuenta ajena ${ }^{6}$. Por lo cual, se cumplirían las notas de laboralidad contempladas en el artículo 1.1 del Estatuto de los Trabajadores (de ahora en adelante ET) -voluntariedad, ajenidad, personal, dependencia o subordinación y retribución-.

De manera que, para poder considerar que se realiza tal función con carácter profesional habrá de acudirse a la nota de laboralidad denominada retribución. Por lo cual si dicho cuidador no tiene tal actividad como su modus vivendi no se le puede encuadrar en la figurar del cuidador profesional de personas en situación de dependencia. Cuestión distinta serían los supuestos de fraude y simulación.

Empero, cabe la posibilidad, a su vez, de que la Administración competente autorice que el cuidador no profesional sea otra persona diferente de los casos excepcionales del apartado 3 del artículo 12 de Real Decreto 1051/2013 de 27 de diciembre, por el que se regulan las prestaciones del Sistema para la Autonomía y Atención a la Dependencia, establecidas en la Ley 39/2006, de 14 de diciembre, de Promoción de la Autonomía Personal y Atención a las personas en situación de dependencia (de ahora en adelante RD 1051/2013). Así, cuando la persona en situación de dependencia tenga su domicilio en un

\footnotetext{
${ }^{4}$ MARTÍN VALES, Priscila, "La recuperación de la financiación pública de las cuotas correspondientes al convenio especial de los cuidadores no profesionales de personas en situación de dependencia", Trabajo y Derecho: nueva revista de actualidad y relaciones laborales, 2019, n 58, p. 101.

5 AYALA DEL PINO, Cristina, "La importancia del cuidado: el cuidador informa, los empleados del servicio doméstico y cuidadores domésticos internos", Anuario jurídico y Económico Escurialense, 2020, no 53 , p. 17.

${ }^{6}$ SELMA PEÑALVA, Alejandra, "La (des)protección social de los cuidadores no profesionales", Revista Derecho social y empresa, 2019, $\mathrm{n}^{\circ} 10$, p. 8.
} 
entorno con insuficiencia de recursos acreditados, despoblado o con circunstancias que impidan o dificulten otras modalidades de atención, la Administración competente está facultada para, de forma excepcional, permitir la existencia de cuidados no profesionales por una persona de su entorno que, aun no teniendo grado de parentesco, resida en el municipio de la persona dependiente o en uno vecino y lo haya hecho durante el período de un año previo a la solicitud ${ }^{7}$. En dichos supuestos al cuidador no profesional se le denomina «cuidador del entorno ${ }^{8} »$.

No obstante, estos criterios adolecen de ambigüedades y falta de concreción para la determinación y aplicación de los mismos, por lo cual, como posible solución para paliar las mismas se podría instar al legislador a la concreción de dichos parámetros jurídicos indeterminados, todo ello, con la finalidad última de que tal figura del cuidador del entorno fuera más eficaz y fácilmente aplicable.

Cuando el cuidador o cuidadora no profesional y la persona en situación de dependencia les une un vínculo de pareja de hecho, tutores y personas designadas, administrativa o judicialmente, con funciones de acogimiento, se considerarán «situaciones asimiladas a la relación familiar» ${ }^{9}$.

Sin embargo, el artículo 2 del Real Decreto 1620/2011, de 14 de noviembre por el que se regula laboral de carácter especial del servicio del hogar familiar, prohíbe expresamente que la persona que asuma la figura del cuidador no profesional desempeñe simultáneamente la condición de empleado del hogar. A lo que se le une la idoneidad física y psíquica para la función del cuidador no profesional, contar con la disponibilidad necesaria para poder llevar a cabo los cuidados demandados por la persona en situación de dependencia; designación de una persona cuidadora principal, la cual asume la responsabilidad de los cuidados durante un periodo mínimo de un año, pudiendo estar auxiliada por cuidadores complementarios ${ }^{10}$.

Por otro lado, a través de los trabajos colaborativos se puede llegar a satisfacer cuantiosas necesidades sociales. Siendo esta una forma moderna de trueque, en la que ninguna de las partes ostenta la condición clásica de empresario ni la de persona trabajadora. Cuestión de difícil enmarque regulatorio y en consecuencia, del disfrute de derechos sociales derivados de tal condición.

Nos obstante, es relevante traer a colación que la prestación de cuidados por un no profesional no está remunerada, ni se ajusta a un horario, ni permite descansos o

\footnotetext{
${ }^{7}$ MARTÍN VALES, Priscila, "La recuperación de la financiación pública de las cuotas correspondientes al convenio especial de los cuidadores no profesionales de personas en situación de dependencia", Trabajo y Derecho: nueva revista de actualidad y relaciones laborales, 2019, nº 58, p. 102.

${ }^{8}$ GONZÁLEZ DE PATTO, Rosa $\mathrm{M}^{\mathrm{a}}$, "La reforma de la protección social de los cuidadores no profesionales de las personas en situación de dependencia. Retrocesos e incertidumbres", Revista Doctrinal Aranzadi Social, 2014, n⿳ 11 .

${ }^{9}$ GONZÁLEZ DE PATTO, Rosa Ma " "La reforma de la protección social de los cuidadores no profesionales de las personas en situación de dependencia. Retrocesos e incertidumbres”, Revista Doctrinal Aranzadi Social, 2014, n ${ }^{\circ} 11$, p. 5.

${ }^{10}$ Acuerdo del Consejo Territorial de 25 de enero de 2010.
} 
vacaciones, por señalar algunos ejemplos de la manifestación de la fáctica desprotección social de este colectivo.

Ello no es óbice, para la aparición de la corriente doctrinal que considera que la suma de la compensación económica, la cobertura de la Seguridad Social y la formación profesional, apuntaba a una «laboralización» ${ }^{11}$ de la figura del cuidador no profesional, de carácter familiar. Cuestión compartida por esta parte.

Por otro lado, la doctrina judicial ${ }^{12}$ ha considerado que sobre la figura de los cuidadores no profesionales se puede concretar en los siguientes aspectos: el carácter no laboral de la relación entre la persona en situación de dependencia y el cuidador no profesional; el requisito de convivencia entre ambos; los efectos del convenio especial; la compatibilidad con otras prestaciones, $y$, la fecha de efectos del reconocimiento de la prestación.

El carácter no laboral de la relación de cuidado entre la persona en situación de dependencia y el cuidador no profesional en el entorno laboral, excluye los supuestos en los que pueda concederse una autorización de residencia temporal y trabajo de un extranjero por la prestación de servicios de ciudadanos en el entorno familiar ${ }^{13}$. No constituyendo despido la resolución administrativa nombrando a un nuevo cuidador no profesional $^{14}$, así como tampoco tiene tal consideración el cese de los servicios debido al fallecimiento de la persona en situación de dependencia ${ }^{15}$.

En referencia al requisito de convivencia entre el cuidador no profesional y la persona en situación de dependencia, se ha entendido que el empadronamiento posee un valor de presunción de residencia, el cual pude verse desvirtuado por la aportación de pruebas en su contra. Así mismo, también se considera prueba de convivencia el que el cuidador no profesional en la misma planta que la persona en situación de dependencia ${ }^{16}$. Por lo cual, en los supuestos en los exista un interrupción de la convivencia, se extinguiría la prestación $^{17}$.

El Tribunal Supremo, mediante su sentencia de la Sala de lo social, Sección 1a , de 11 de julio de 2014, ha señalado que ha de tenerse en cuenta para el cálculo de la prestación por incapacidad permanente para la profesión habitual, las cotizaciones del convenio

11 GONZÁlEZ DE PATTO, Rosa Ma, "La reforma de la protección social de los cuidadores no profesionales de las personas en situación de dependencia. Retrocesos e incertidumbres", Revista Doctrinal Aranzadi Social, 2014, n 11, p. 3.

12 Ver Sentencias del Tribunal superior de Justicia de Murcia, Sala de lo contencioso-administrativo, Sección 1a , de 1 de diciembre de 2017; Madrid, Sala de lo contencioso-administrativo, Sección ${ }^{\text {a }}$, de 14 de julio de 2016.

${ }^{13}$ Sentencia del tribunal superior de Justicia de Andalucía, Málaga, sala de lo contencioso-administrativos, Sección $3^{\mathrm{a}}$, de 20 de noviembre de 2015.

${ }^{14}$ Sentencia del Tribunal Superior de Justicia, Valencia, Sala de lo Social, Sección 1 ${ }^{\text {a }}$, de 3 de abril de 2013.

${ }^{15}$ Sentencia del Tribunal superior de justicia de Murcia, Sala de lo Social, Sección $1^{\text {a }}$, de 11 de julio de 2016.

${ }^{16}$ Sentencia del Tribunal Superior de Justicia, País Vasco, Sala de lo contencioso-administrativo, Sección $8^{\mathrm{a}}$, de 9 de julio de 2014.

${ }^{17}$ Sentencias del Tribunal Superior de Justicia de La Rioja, Sala de lo contencioso-administrativo, Sección $1^{\mathrm{a}}$, de 27 de junio de 2013. 
especial $^{18}$. Con respecto a la compatibilidad con otras prestaciones, la doctrina judicial se encuentra dividida.

No obstante, el punto donde se ha concentrado la mayor litigiosidad de la figura del cuidador no profesional es la fecha de efectos del reconocimiento de la prestación, por lo cual, los efectos de la prestación económica deben reconocerse desde el día siguiente a la fecha de presentación de la solicitud por la persona en situación de dependencia ${ }^{19}$-la beneficiaria-. Es importante traer a colación que el fallecimiento de la persona en situación de dependencia y, por ende, el beneficiario de la prestación, no extingue el derecho a una prestación economice ya generada por el reconocimiento de la situación de dependencia y el inicio de la prestación correspondiente ${ }^{20}$.

Sin embargo, el retraso de las resoluciones por parte de la Administración del reconocimiento del derecho ha conllevado inexorablemente a que los juzgadores establezcan los efectos de la misma desde su reconocimiento.

No obstante, junto con este medidas existen otras de contenido civil en complemento de las primeras, en concreto, en las funciones del cuidador no profesional en la esfera personal y patrimonial de las personas dependientes ${ }^{21}$. Por lo que, el criterio de la idoneidad en la designación de cargos tutelados, puede verse en el requisito de capacitación del cuidador no profesional contemplado por las Comunidades Autónomas.

\section{Mecanismos de protección de los cuidadores no profesionales de las personas en situación de dependencia}

\section{Recuperación de la financiación pública}

Tres son las modalidades de prestaciones económicas que se contemplan en la Ley 39/2006: la prestación económica vinculada al servicio -artículo 17-, la prestación económica para los cuidados del entorno familiar y apoyo a los cuidadores no profesionales -artículo 18-, y la prestación económica de asistencia personal -artículo 19-

\footnotetext{
${ }^{18}$ En el mismo sentido se ha pronunciado el Tribunal Superior de Justicia, Sala de lo Social, en su sentencia de 1 de octubre de 2014.

${ }^{19}$ Sentencia del Tribunal Superior de Justicia de Cataluña, Sala de lo Social, Sección $1^{\text {a }}$, de 22 de octubre de 2010. Sentencias del Tribunal Superior de Justicia de Valencia, Sala de lo contencioso-administrativo, Sección 5a, de 9 de noviembre de 2010; de 17 de noviembre de 2010, de 5 de julio de 2011, de 6 de septiembre de 2011, de 15 de noviembre de 2011, de 9 de mayo de 2012 y de 30 de noviembre de 2012. Sentencia del Tribunal Superior de Justicia de Islas Baleares, Sala de lo contencioso-administrativo, Sección 1 $1^{\text {a }}$, de 4 de abril de 2011. Sentencia del Tribunal Superior de Justicia de Murcia, Sala de lo contencioso-administrativo, Sección $1^{a}$, de 25 de enero de 2013. Sentencias del Tribunal Superior de Justicia de Madrid, Sala de lo Contencioso-administrativo, Sección $8^{\mathrm{a}}$, de 15 de abril de 2014, entre otras.

${ }^{20}$ Sentencias del Tribunal de Justicia de Valencia, Sala de lo contencioso-administrativo, Sección $4^{\text {a }}$ de 21 de enero de 2015; de 29 de abril de 2015.

FERNÁNDEZ COLLADOS, $\mathrm{M}^{\mathrm{a}}$ Belén, "Los cuidados del entorno familiar más de una década después: los vaivenes en su regulación, su aplicación por la doctrina judicial y sus retos en materia de igualdad", Revista Española de Derecho del Trabajo, 2018, nº 210.

${ }^{21}$ ALBIEZ DOHRMANN, Klaus Jochen, "La asistencia legal de las personas mayores con discapacidad: una institución jurídica complementaria o alternativa", Revista de la Facultad de Derecho de la Universidad de Granada, 2017, nº 16 y 17, p. 14.
} 
22. No obstante, en la mayoría de las comunidades autónomas estas prestaciones se articula a través de sus respectivos Servicios Sociales. Siendo el Consejo Territorial de Servicios sociales y del sistema para la autonomía y Atención a la dependencia, el que establece los criterios de acceso a tales prestaciones, en función del grado de dependencia reconocido por los mismos y su capacidad económica.

La prestación económica para cuidados en el entorno familiar ha sido de las más utilizadas por las personas en situación de dependencia y, así mismo, reconocidas por la Administración al suponer un cargo más reducido en la dotación presupuestaria para el Estado de Bienestar de dichas personas.

Son muchas las formas jurídicas a través de las cuales se pueden desarrollar las tareas de cuidado y atención de una persona que, aun no teniendo la condición formal de dependiente, materialmente gozan de la misma. Pudiendo, en su caso, compaginarlos con otro trabajo remunerado.

Con la Ley 39/2006, el legislador intentó paliar las desigualdades existentes con los cuidadores no profesionales de personas dependientes a través de la suscripción de un convenio con la Seguridad Social, siempre y cuando reúnan una serie de requisitos. Pudiéndose suscribir al mismo tanto aquellos cuidadores no profesionales que dediquen el 100 por ciento de su tiempo en el cuidado de la persona dependiente, como los que realicen dicha actividad de cuidado de forma parcial, complementándolo con una prestación de servicios remunerada a tiempo parcial. No obstante la base de cotización es muy reducida, salvo en los supuestos en los que se pueda mantener la base de cotización anterior -artículo 4.2 del Real Decreto 615/2007, de 11 de mayo, por el que se regula la Seguridad Social de los cuidadores de las personas en situación de dependencia (de ahora en adelante RD 615/2007)-.

Por otro lado, en situaciones excepcionales, la persona dependencia, en virtud de los artículos 14.4 y 18 de la Ley 39/2006, puedan ser beneficiarios de una prestación económica para poder ser atendido por dichos cuidadores no profesionales. De manera que, solamente podrá ser beneficiario de la misma si previamente ha cursado alta y cotización en el régimen general de la Seguridad Social. Lo cual limita enormemente la adquisición de dicha prestación, ya que en la mayoría de los supuestos son personas que no han podido cotizar, bien por haberse dedicado al mantenimiento del hogar, o bien por no haber podido realizar prestación laboral alguna.

La base mensual será el «tope mínimo que, en cada momento, esté establecido en el Régimen general de la Seguridad», así como el modelo de cotización propio de dicho convenio se regulará en el artículo 4 del RD 615/2007. Además de poder verse reducida la misma en los supuestos en los que el cuidador no profesional realizara tal actividad de forma no completa, salvo en los supuestos en los que dicha base sea inferior al 50 por ciento del tope mínimo establecido en el Régimen General. A lo que hay que añadir la

\footnotetext{
${ }^{22}$ FERNÁNDEZ COLLADOS, Ma Belén, "Los cuidados del entorno familiar más de una década después: los vaivenes en su regulación, su aplicación por la doctrina judicial y sus retos en materia de igualdad", Revista Española de Derecho del Trabajo, 2018, n 210, p. 2.
} 
modificación introducida por el RD 175/2011, por la cual, en los supuestos en los que la persona dependiente ostente el grado I, es decir, dependencia moderada, la base se cotización del convenio especial de los cuidadores no profesionales será del 50 por ciento del tope mínimo establecido para el Régimen General de la Seguridad Social.

No obstante, en los supuestos en los que el cuidador no profesional haya interrumpido su actividad profesional -tanto por cuenta propia, como por cuenta ajena-, por la que estaba incluido en el sistema de la Seguridad Social, a razón del comienzo del ejercicio de dicha actividad no remunerada de cuidado, el legislador nacional prevé que este pueda mantener la base de cotización del último ejercicio en tal actividad remunerada, siempre que resulte superior al tope mínimo contemplado en el Régimen General de la Seguridad Social, siendo a su cargo directo el coste del incremento de cotización sobre la cuantía resultante (artículo 4.2 RD 615/2007).

La figura del convenio especial con la Administración de la Seguridad Social no nace, por supuesto, con el RD 615/2007, sino que ve la luz con la Orden TAS/2865/2003, de 13 de octubre, por la que se regula el convenio especial en el sistema de la seguridad social, el cual ha sido modificado en siete ocasiones, siendo la última de ellas la aprobada por la Orden TMS/397/2019, de 4 de abril, que actualiza a la legislación vigente laboral y de Seguridad Social el convenio especial a suscribir en procedimientos de despido colectivo que incluyan a trabajadores con un mínimo de 55 años. Empero, es de notoria importancia traer a colación que la Orden TAS/2865/2003 goza de aplicabilidad supletoria en lo no previsto en el RD 615/2007, en virtud del precepto 5 de tal norma.

Uno de los mayores problemas a los que se enfrentan los cuidadores no profesionales de personas dependientes, es la adquisición de estos últimos del grado discapacidad. De manera que, en los supuestos en los que la persona fáticamente dependiente, empero no formalmente, necesite cuidados y estos sean asumidos por un familiar, en la mayoría de dichos supuestos, el cuidador no profesional de facto no podrá beneficiarse de lo contemplando en la Ley 39/2006. Debido a que, en la mayoría de los supuestos, el grado de dependencia de estas personas no es muy elevado, ya que, los cuidadores no profesionales «no poseen titulación técnica alguna y cuya relación de cercanía y confianza es lo único que les habilita para llevar a cabo el cuidado ${ }^{23} \gg$.

Otra problemática la encontramos en la desprotección social del cuidador derivada del derecho a la intimidad del domicilio familiar en el que se prestan los servicios ${ }^{24}$. Ya que la Inspección de Trabajo y Seguridad Social solo podrá realizar su función de vigilancia de control en estos supuestos, previa autorización del titular de la vivienda o autorización judicial.

Sin embargo, no es hasta el Real Decreto 615/2007, donde se establece la obligatoriedad de inclusión de los cuidadores no profesionales en el campo de aplicación del régimen

\footnotetext{
${ }^{23}$ AYALA DEL PINO, Cristina, "La importancia del cuidado: el cuidador informa, los empleados del servicio doméstico y cuidadores domésticos internos", Anuario jurídico y Económico Escurialense, 2020, n 53 , p. 14.

${ }^{24}$ SELMA PEÑALVA, Alejandra, "La (des)protección social de los cuidadores no profesionales", Revista Derecho social y empresa, 2019, ${ }^{\circ}$ 10, p. 18.
} 
general de la Seguridad Social, asimilando su situación a la del alta ${ }^{25}$, mediante la suscripción de un convenio especial, para el cual no será necesario un periodo previo de cotización (artículo 2.1).

Así mismo dicha norma prevé la posibilidad de que una persona trabajadora reduzca su jornada, con la correspondiente reducción de retribución, a favor de la adquisición de condición de cuidador no profesional. En tales supuestos, la suma de la base de cotización del cuidador no profesional, no podrá superar a la base por la que se venía cotizando antes de reducir la jornada y la correspondiente retribución, como consecuencia de la atención familiar de la persona en situación de dependencia (artículo 4.2 RD 615/2007). Cotizándose, además, en la cantidad de un 0,2 por ciento de la base de cotización en concepto de formación profesional.

El RD 20/2012 con su disposición Adicional 2a, eliminó el efecto retroactivo de la prestación económica para cuidados en el entorno familiar y apoyo a cuidadores no profesionales. Además, mediante dicha norma la inclusión de los cuidadores no profesionales en el Régimen General de la Seguridad Social deja de tener un cariz obligatorio para ser voluntario. Lo cual se acentúa con la Disposición transitoria $13^{\mathrm{a}}$, la cual expresa que los convenios preexistentes a la entrada en vigor del RD 20/2012, quedarán extinguidos el día 31 de agosto de 2012, salvo que el cuidador no profesional hubiera solicitado expresamente el mantenimiento del mismo con anterioridad al 2 de noviembre de 2012, en cuyo caso se entenderá que subsiste desde el día 1 de septiembre de 2012. Así mismo, dicha cotización correrá a cargo de la tesorería General de la Seguridad Social y comenzará a recaer en el propio cuidador no profesional. Haciéndose efectiva tal medida a partir del 1 de enero de 2013 y estableciendo que a partir del 1 de septiembre de 2013 y hasta el 31 de diciembre de 2012, la cotización a la Seguridad Social de los cuidadores no profesionales tendrá una reducción del 10 por ciento en el total de la cuota a abonar, siendo sufragada el 5 por ciento a cargo de la Administración General del Estado y el 85 por ciento restante a cargo del cuidador no profesional.

Aunque desde la entrada en vigor del RD 615/2007, la aportación de la cuantía de las cotizaciones de los cuidadores no profesionales correspondía a la Administración General del Estado, esta situación cambio con el RD 20/2012. Además, mediante esta última norma, en su Disposición Transitoria Décima reduce hasta un 15 por ciento las cuantías máximas recibidas por los cuidados en el entorno familiar. Lo cual puede conllevar a una clara voluntad de desincentivar las actividades de cuidados en el entorno familiar ${ }^{26}$.

Dicho cambio de régimen de la Seguridad Social podría considerarse contrario al principio de igualdad ${ }^{27}$ recogido en el artículo 14 de nuestra norma suprema, a razón del

\footnotetext{
${ }^{25}$ Ver Sentencia del Tribunal Superior de Justicia, Murcia, Sala de lo contencioso-administrativo, Sección $1^{\mathrm{a}}$, de 28 de junio de 2019.

${ }^{26}$ FERNÁNDEZ COLLADOS, M Melén, "Los cuidados del entorno familiar más de una década después: los vaivenes en su regulación, su aplicación por la doctrina judicial y sus retos en materia de igualdad", Revista Española de Derecho del Trabajo, 2018, $\mathrm{n}^{\circ} 210$.

${ }^{27}$ GONZÁlEZ DE PATTO, Rosa $\mathrm{M}^{\mathrm{a}}$, "La reforma de la protección social de los cuidadores no profesionales de las personas en situación de dependencia. Retrocesos e incertidumbres", Revista Doctrinal Aranzadi Social, 2014, $\mathrm{n}^{\circ} 11$.
} 
trato indiscriminado de la carga de cotización del cuidador no profesional, no teniendo en consideración sus ingresos y respectivas cargas familiares, lo cual conllevaría, a su vez, a una discriminación debida a su situación social y capacidad económica. Siendo de notoria importancia la creación por parte del legislador de medidas correctoras para conseguir la tan buscada equidad e igualdad efectiva.

Por otro lado, el RD 615/2007, establece la aplicación de programas y acciones formativas a los cuidadores no profesionales y prevé su financiación mediante un tipo reducido de su cotización. Dicho Real Decreto, desarrollado por la Orden TAS/718/2008, de 7 marzo, y posteriormente por la Resolución de 4 de noviembre de 2009, de la Secretaría General de Política Social y Consumo, por la que se publica el Acuerdo del Consejo Territorial del SAAD, sobre objetivos y contenidos comunes del Sistema de información del SAAD que determina los criterios comunes de acreditación en materia de formación e información de los cuidadores no profesionales. Por lo cual, serán las respectivas administraciones autonómicas las encargadas de establecer, en cada caso, los criterios para determinar las necesidades formativas de los cuidadores no profesionales destinatarias de las acciones formativas en función de su perfil ${ }^{28}$.

Las áreas básicas de formación inicial para los cuidadores no profesionales se contemplan en las siguientes: la consideración del cuidador no profesional; medidas higiénico sanitarias; grupos de autoayuda y ayuda mutua; competencias y habilidades para el cuidado; cuidados sanitarios y psicosociales; técnicas para favorecer la libre determinación y la máxima autonomía individual de la persona en situación de dependencia; técnicas para favorecer las relaciones sociales; actividades de acompañamiento y de relación social; estrategias de intervención; ocio y tiempo libre de la persona en situación de dependencia; recursos existentes y generación de redes sociales e información ${ }^{29}$.

En cuanto a los periodos de descanso, no existe en términos equiparables al del cuidador profesional, únicamente dejando el legislador a las Comunidades Autónomas la forma de promover acciones de apoyo a los cuidadores no profesionales para que puedan disfrutar de periodos de descanso. Como por ejemplo, en la Comunidad Autónoma de Galicia, la cual fue condenada por el Tribunal Superior de Justicia de dicha Comunidad Autónoma ha aprobar el PIA ${ }^{30}$, así como a aprobar el «contenido de la libranza para cuidados en el

\footnotetext{
28 GONZÁLEZ DE PATTO, Rosa M" "La reforma de la protección social de los cuidadores no profesionales de las personas en situación de dependencia. Retrocesos e incertidumbres", Revista Doctrinal Aranzadi Social, 2014, n 11, p. 18.

${ }^{29}$ GONZÁlEZ DE PATTO, Rosa $\mathrm{M}^{\mathrm{a}}$, "La reforma de la protección social de los cuidadores no profesionales de las personas en situación de dependencia. Retrocesos e incertidumbres", Revista Doctrinal Aranzadi Social, 2014, n 11, p. 13.

30 El Programa Individual de Atención (PIA), es un informe elaborado por los Servicios Sociales correspondientes al municipio de la persona en situación de dependencia, por el cual se recogen las modalidades de intervención más adecuadas a la persona en función de los recursos previstos en la resolución para su grado y nivel.
} 
entorno familiar a prestar por su madre, como cuidadora no profesional, y con eficacia retroactiva al 1 de abril de $2009^{31} \gg$.

\section{Mecanismos de conciliación y flexibilización}

Una de las medidas a impulsar para favorecer la inserción en el mercado laboral de los cuidadores no profesionales, pudiendo compaginar tal actividad con una prestación de servicios, tanto por cuenta propia como por cuenta ajena, remunerada, serían las denominadas medidas de flexibilización.

No obstante, para los supuestos en los que la persona dependiente lo sea de manera temporal, el ET prevé la concesión por parte del empresario de un permiso retribuido de dos días en los supuestos de accidente grave con el que el cuidador no profesional y trabajador posea vínculos de consanguinidad o afectividad de hasta el segundo grado, así como, en los supuestos de enfermedad grave, hospitalización o intervención quirúrgica sin hospitalización.

Siendo estas medidas, en la mayoría de los supuestos, insuficientes, debido a que la situación de dependencia de prolonga en el tiempo, más allá de los parámetros citados anteriormente. Por lo cual, otro mecanismo que podría usar el trabajador es la excedencia, la cual implica una suspensión de empleo y sueldo que no deja de ser perjudicial para el mismo, ya que con ella, pierde todos los beneficios sociales y económicos que venía disfrutando por ser trabajador.

No obstante, el disfrute de dicho mecanismo de flexibilización y conciliación es limitado en el tiempo y se encuentra vinculado únicamente por razones de parentesco entre el cuidador no profesional y la persona en situación de dependencia. A falta de ampliación del derecho, el artículo 37.3 ET, adolece de una notable rigidez, no solo con la consideración del vínculo entre el cuidador no profesional y la persona en situación de dependencia, sino con la horquilla temporal en la que puede ser el mismo disfrutado, no subordinando la «necesidad de atención que pueda generar el cuidado del enfermo o accidentado $^{32} \gg$. En consecuencia, para que esta medida fuera eficaz y lo menos lesiva para los cuidadores no profesionales, el legislador debería proceder a su revisión para hacerla más flexible y, en su defecto, dotar de las armas necesarias para que mediante la negociación colectiva pudiera dotar de dicha flexibilidad a este tipo de excedencias mediante los convenios o acuerdos colectivos aplicables.

En los supuestos en los que la persona dependiente lo fuera a raíz de su avanzada edad mayor de 65 años-, únicamente el cuidador no profesional tendría que justificar que la persona en situación de dependencia de avanzada edad soporta una imposibilidad de valerse por sí mismo a causa del envejecimiento. Por lo cual, en este supuesto podemos observar que se da la circunstancia contraria que en el caso precedente, la regulación del

\footnotetext{
${ }^{31}$ Sentencia del Tribunal Superior de Justicia, Sala de lo contencioso-administrativo, Sección $1^{\text {a }}$, de 5 de junio de 2019, fundamento de derecho $3^{\circ}$.

32 VILLABA SÁNCHEZ, Alicia, "Los derechos de los trabajadores con personas de avanzada edad a su cargo. Una aproximación al cuidado de personas de avanzada edad desde el Derecho del Trabajo Español", Revista Española de Derecho del Trabajo, 2017, n 195, p. 10.
} 
mismo posee una excesiva flexibilización que dificulta su posible previsión de resultado en procedimientos judiciales y extrajudiciales derivados de la negativa del empresario a la protección de concesión de dicho derecho de la persona trabajadora.

Cuando la relación de parentesco entre el cuidador no profesional y la persona en situación de dependencia sea inexistente o no sea la establecida en el precepto, le quedará la opción al primero de acudir a la excedencia voluntaria. Empero tal modalidad de excedencia es considerablemente menos garantista para el trabajador y, a su vez, cuidador no profesional.

Por otro lado, el legislador nacional ha plasmado en el ET unas medidas de carácter indefinido -su duración no está sometida a la conclusión de un periodo cierto-, las cuales son, relativamente, de reciente creación. De manera que, en primer lugar, los cuidadores no profesionales que realicen una prestación de servicios por cuenta ajena de carácter remunerada, podrán adaptar la jornada en virtud de las necesidades de cuidado de la persona en situación de dependencia que esté a su cargo. No necesitando, por ende, la separación de la persona trabajadora del mercado laboral para realizar la labor de cuidado. Empero, este derecho no se encuentra desarrollado en la norma común, sino que habría que acudir a los convenios y acuerdos colectivos aplicables a cada empresa y sector, para poder observar si se reconoce o tal derecho ${ }^{33}$. Por lo cual, solo podrán beneficiarse de tal derecho los trabajadores que en su convenio colectivo se encuentre recogido. Lo cual nos lleva a la premisa de que en la mayoría de las ocasiones las PYMES y microempresas, así como los autónomos con asalariados, no gozan de una negociación colectiva que recoja este derecho.

La negociación colectiva supra empresarial no recoge la determinación y desarrollo de este tipo de derechos, dejándoselos al desarrollo de aquellos convenios o acuerdos colectivos cuyo ámbito de aplicación sea la empresa. A lo que hay que añadir que nuestro tejido empresarial lo forman en su inmensa mayoría PYMES, microempresas y autónomos con asalariados, superando el 90 por ciento de las mismas. Lo cual nos deja un número residual de empresas en las que se puede introducir y establecer los criterios de aplicación del derecho a la modulación de la jornada por la necesidad de cuidado de una persona en situación de dependencia a su cargo. Siendo, por ende, la efectividad de esta medida muy reducida.

Otra de las medidas de flexibilización y conciliación de carácter indefinido es la denominada reducción de jornada de trabajo. Para poder acogerse a dicha medida, el trabajador y cuidador no profesional ha de mantener un vínculo de consanguinidad o afinidad con la persona en situación de dependencia de hasta el segundo grado, que por razones de accidente o enfermedad no pueda valerse por sí mismo y que, a su vez, este último no desempeñe actividad tribuida alguna. No obstante, podemos observar que quedan fuera de tal supuesto aquellas personas en situación de dependencia que lo sean por enfermedad o envejecimiento, lo cual soslaya la aplicación de dicha medida.

\footnotetext{
${ }^{33}$ El artículo 34.8 ET contempla el derecho de la persona trabajadora a adaptar la duración y distribución de su jornada en aras de hacer efectivo su derecho a la conciliación de la vida laboral, personal y familiar, sin embargo, deja su desarrollo y aplicación a la negociación colectiva.
} 
Como tercera medida de carácter indefinido tenemos el derecho del traslado de la persona trabajadora a razón del domicilio de su núcleo familiar. El ET reconoce este derecho a aquellas personas trabajadoras que se vean obligadas a abandonar su localidad por su condición de víctimas de violencia de género o victimas del terrorismo, así como, además, a aquellas que posean un grado de dependencia que lo justifique. De manera que, siempre y cuando una vacante en la empresa del mismo grupo profesional en otra localidad, tendrá derecho de preferencia a ocuparla las personas trabajadoras anteriormente mencionadas.

Por lo cual, queda al arbitrio de la negociación colectiva los supuestos en los que las personas trabajadoras y cuidadoras no profesionales puedan acogerse a este derecho.

\section{Aumento de la desprotección social tras el inicio de la pandemia Covid-19}

La inexistencia de un sistema institucional de promoción de la autonomía personal y atención a las personas en situación de dependencia, así como las insuficiencias y carencias que hasta en la actualidad se están dando y que se están agravando enormemente debido a la crisis provocada por el Covid-19, se han ido asumiendo e intentado mitigar por los familiares. Tal asunción y su consiguiente sacrificio personal y laboral ha incido en mayor medida en las mujeres, debido a los roles de cuidado tan arraigados en la actualidad, viéndose desplazados a la hora de adquirir derechos en materia de Seguridad Social. Por lo que, se hace necesario, con carácter de urgencia, la configuración de la prestación económica del cuidador no profesional, o en su caso, de la reestructuración del convenio con la Seguridad social de carácter gratuito consagrado en el Real Decreto $615 / 2007$.

Siendo esencial la determinación de si el cuidado de la persona dependiente se realiza de forma no profesional, debido a las situaciones de fraude y simulación de relaciones altruistas o de buena vecindad, cuando en realidad son relaciones de prestación de servicios de cuidados del cuidador hacia la persona con dependencia. Y, por ende, no se declara la retribución monetaria o en especie recibido por esta, favoreciendo, con ello, la economía sumergida.

Es de notoria relevancia traer a colación que la promoción del bienestar social, incluyendo a las personas dependientes, es un principio rector de la política económica y social ${ }^{34}$ consagrado en nuestra norma suprema -art. $50 \mathrm{CE}$-.

Con la entrada en vigor del Real Decreto-ley 6/2019, de 1 de marzo, de medidas urgentes para la garantía de igualdad de trato entre mujeres y hombres en el empleo y la ocupación, se incluyó, mediante su exposición de motivos, «una medida de protección social de carácter extraordinario y urgente, como es la recuperación de la financiación de las cuotas del convenio especial de los cuidadores no profesionales de las personas en situación de

\footnotetext{
${ }^{34}$ VILLABA SÁNCHEZ, Alicia, "Los derechos de los trabajadores con personas de avanzada edad a su cargo. Una aproximación al cuidado de personas de avanzada edad desde el Derecho del Trabajo Español", Revista Española de Derecho del Trabajo, 2017, nº 195, p. 4 y ss.

Ver Sentencia del Tribunal Constitucional 222/1992, de 11 de diciembre (BOE núm. 16, de 19 de enero de 1993).
} 
dependencia a cargo de la Administración General del Estado. De esta forma, se pone en valor la figura del cuidador no profesional de personas en situación de dependencia, quien en muchos casos se ve abocado a abandonar su puesto de trabajo, y por tanto a interrumpir su carrera de cotización a la seguridad social, para cuidar de la persona dependiente. Esta medida, dada su particular naturaleza, requiere de una actuación urgente, y sin duda necesaria para este colectivo, que está formado en mayor medida por mujeres, ya que tradicionalmente son ellas las que asumen los cuidados de las personas dependientes».

Dicha norma intentó dar visibilidad social a los cuidadores no profesionales, intentando, por ende, paliar las dificultades de este colectivo. No obstante, se quedó en una mera declaración de intenciones. En primer lugar, por la no recuperación de la financiación pública de la cotización y valoración del convenio especial ${ }^{35}$. Y, en segundo lugar, por la posterior crisis sanitaria, y en la cual continuamos inmersos, que dio lugar a dos estados de alarma consecutivos -el primero comprendido entre los meses de marzo a junio de 2020 y el segundo en el que nos encontramos inmersos desde septiembre-.

Anteriormente a la crisis derivada del covid-19, la línea jurídica de separación entre un cuidador no profesional de personas en situación de dependencia y un trabajador doméstico se encontraba delimitada con una mayor concreción. Siendo los primeros, en su mayoría, parientes, consanguíneos o no, de las personas necesarias de cuidados, mientras que, los segundos no se relacionan con los sujetos de cuidado mediante el parentesco. Además, de la existencia de una regulación diferencia, pero conexa en algunos extremos.

Después de la declaración del estado de alarma, este sector se vio claramente afectado, al igual que muchos otros, empero no con carácter negativo. De manera que, el número de usuarios -personas dependientes-, que han solicitado los servicios de cuidador profesional y no profesional, en su caso, se ha visto incrementado notablemente.

Al cese del estado de alarma, se ha producido un cambio a la hora de entender las relaciones laborales de cuidado, aumentando la oferta de cuidadores a domicilio en detrimento de la demanda de plazas en residencias. A esta situación hay que añadir el aumento de la tasa de paro en la actualidad, la cual, lamentablemente, está tendiendo al alta. Convirtiéndose, por ende, el sector de los cuidados en el domicilio del usuario, en un sector al alza en nuestro país. Por lo que deberá de ser objeto de una amplia regulación, a raíz de la escasa regulación actual en dicho sector, delimitándose, con ello, las figuras del cuidador no profesional y del cuidador profesional.

A lo que hay que añadir el agravio comparativo que sufren los cuidadores no profesionales de personas en situación de dependencia en relación con aquellas personas que disfrutan del derecho a la excedencia por cuidado de hijo a cargo. De manera que, en virtud del artículo 237 Ley General de Seguridad Social, a estos últimos se les computará el primer año de excedencia a efectos del cómputo de periodos de carencia a la hora de

\footnotetext{
${ }^{35}$ MARTÍN VALES, Priscila, "La recuperación de la financiación pública de las cuotas correspondientes al convenio especial de los cuidadores no profesionales de personas en situación de dependencia", Trabajo y Derecho: nueva revista de actualidad y relaciones laborales, 2019, nº 58, p. 105.
} 
determinar la concesión y correspondiente cuantía de prestaciones, tales como la jubilación, incapacidad permanente, muerte y supervivencia, maternidad y paternidad. Empero, quedarán fuera de la acción protectora la incapacidad temporal, teniendo como uno de los focos de vicisitudes la calificación de las contingencias profesionales que surgen en el cuidador familiar ${ }^{36}$.

Es de necesaria concreción y de notoria urgencia la elaboración de mecanismos y regulación, en su caso, por los que deje de considerarse a la figura del cuidador no profesional como algo residual y extraordinario y comience, por ende, a normalizarse dicha actividad de cuidado en la persona del trabajador, visibilizando dicha actividad.

El cuidador no profesional -o cuidadora en la mayoría de los casos-, lleva a cabo una actividad de cuidado esencial en nuestra sociedad, empero con la reforma llevada a cabo en 2012 debido a crisis económica, junto con la crisis sanitaria provocada con el Covid19, la cual ha llevado consigo una crisis económica y humanitaria sin precedentes, ha puesto en jaque al Estado del Bienestar y a la igualdad entre mujeres y hombres. Siendo ahora más necesario que nunca volver a los parámetros contemplados en la Ley 39/2006, de manera que volviera a ser considerada obligatoria la cotización a la Seguridad Social de los cuidadores no profesionales, siendo estas sufragadas por la Administración. Es decir, aumentar la inversión pública en los cuidadores no profesionales y las personas en situación de dependencia, así como en última instancia, en el Estado de Bienestar.

Por lo cual, para la pervivencia del Estado de Bienestar, la norma ha de estar en continuo cambio, con la finalidad de adecuar al mismo a los cambios sociales y económicos, para lo cual será necesario la incorporación de «nuevas respuestas a situaciones cada vez más complejas de integración y pertenencia al grupo social organizado ${ }^{37} \gg$.

En su defecto, podría establecerse una bonificación de la cuota de los cuidadores no profesionales de las personas en situación de dependencia, cuando los primeros tuvieran 45 o más años. Ya que este tipo de trabajadores son los que mercado laboral rechaza pese a su amplia experiencia y sapiencia en el sector.

\section{Bibliografía}

ALBIEZ DOHRMANN, Klaus Jochen, "La asistencia legal de las personas mayores con discapacidad: una institución jurídica complementaria o alternativa", Revista de la Facultad de Derecho de la Universidad de Granada, 2017, nº 16 y 17.

AYALA DEL PINO, Cristina, "La importancia del cuidado: el cuidador informa, los empleados del servicio doméstico y cuidadores domésticos internos", Anuario jurídico y Económico Escurialense, 2020, nº 53, p. 139-164.

36 GONZÁlEZ DE PATTO, Rosa $\mathrm{M}^{\mathrm{a}}$, "La reforma de la protección social de los cuidadores no profesionales de las personas en situación de dependencia. Retrocesos e incertidumbres", Revista Doctrinal Aranzadi Social, 2014, n 11, p. 12.

${ }^{37}$ LÓPEZ MARTÍNEZ, Juan, "La construcción de un sistema integral de protección de las personas con necesidades especiales en un contexto de crisis. Un apunte desde las medidas fiscales", Revista de la Facultad de Derecho de la Universidad de Granada, 2017, nº 16, 17 y 18, p. 4. 
FERNÁNDEZ COLLADOS, Ma Belén, "Los cuidados del entorno familiar más de una década después: los vaivenes en su regulación, su aplicación por la doctrina judicial y sus retos en materia de igualdad", Revista Española de Derecho del Trabajo, 2018, nº 2010.

GONZÁLEZ DE PATTO, Rosa M" "La reforma de la protección social de los cuidadores no profesionales de las personas en situación de dependencia. Retrocesos e incertidumbres", Revista Doctrinal Aranzadi Social, 2014, nº 11.

LÓPEZ MARTÍNEZ, Juan, "La construcción de un sistema integral de protección de las personas con necesidades especiales en un contexto de crisis. Un apunte desde las medidas fiscales", Revista de la Facultad de Derecho de la Universidad de Granada, 2017, no 16,17 y 18.

MARTÍN VALES, Priscila, "La recuperación de la financiación pública de las cuotas correspondientes al convenio especial de los cuidadores no profesionales de personas en situación de dependencia", Trabajo y Derecho: nueva revista de actualidad y relaciones laborales, 2019, nº 58, p. 99-103.

SELMA PEÑALVA, Alejandra, "La (des)protección social de los cuidadores no profesionales", Revista Derecho Social y Empresa, 2019, nº 10, p. 110-134.

VILLABA SÁNCHEZ, Alicia, "Los derechos de los trabajadores con personas de avanzada edad a su cargo. Una aproximación al cuidado de personas de avanzada edad desde el Derecho del Trabajo Español”, Revista Española de Derecho del Trabajo, 2017, $\mathrm{n}^{\mathrm{o}} 195$. 